\title{
The thermal conductance of collection tubes in geothermal energy systems
}

\author{
R. L. Frederick \& A. Zenteno \\ Departamento de Ingeniería Mecánica, Universidad de Chile, Chile
}

\begin{abstract}
The numerical simulation of heat transfer in geothermal heat exchangers is performed. Systems consisting of horizontal tubes embedded in the ground are simulated for time periods of 3 months. Cases comprise OF a single tube, two parallel tubes and a horizontal array of 9 tubes. The thermal conductance between tubes and soil is defined and numerically determined, and scaling laws are formulated for its dependence on the main independent variables. Interference between tubes in arrays is also characterized by an index relating to heat transfer from a single tube. The design criteria for tube arrays is formulated. Keywords: geothermal heat exchangers, thermal conductance, numerical model.
\end{abstract}

\section{Introduction}

Heat pumps are efficient in space heating systems based on low enthalpy energy sources, and are used in many parts of the world. Taking advantage of the stability of soil temperatures, these systems can supply the needs of heating and cooling of houses and public buildings. The heat exchanger coupling the heat pump and the soil is perhaps the most difficult component to design in these systems. One of the main design difficulties is to obtain reliable information on the overall heat transfer coefficients for these exchangers.

In heat exchanger design methods, detailed geometries must be proposed and then rated. Rating requires simulations to predict the long term behaviour of the exchangers, and to describe the seasonal effects on its performance. Several types of heat exchanger are in use at present. These include heat exchangers with tubes in an aquifer, in which water moves at speeds of some centimetres per day, giving rise to some convective heat transfer. Other heat exchangers consist of tubes embedded in the soil. For the latter type, conduction is the dominant heat 
transfer mechanism and one has to consider the wide variability of the relevant physical properties of the soil: thermal conductivity, density and specific heat. For each new project these properties have to be determined specifically for the site. Embedded tubes modify the soil temperature around them, and this effect propagates reaching considerable distances in a long period, such as a season. This may deteriorate the overall heat transfer capability of the system. Recovery of the soil temperature must be made possible between seasons. If not, long term effects may accumulate through the project lifetime. For small applications it has been suggested that a heat transfer rate of 50-60 W/m of tube can be enough information for design. For bigger applications, which require significant tube lengths, multiple tubes or serpentine tubes, the heat transfer deterioration in time can be considerable, and more accurate methods are required. Interference between tubes is also an acute problem in tubes placed in a solid environment.

In response to these problems many analytical and numerical solutions have been developed. The classical analytical solutions are the line source model and the cylindrical source, all of which assume a uniform heat flux at the tube wall [1]. The recent numerical models are very refined and can accommodate for any type of grid geometry that may give great detail of the temperature variation around the pipes and in the ground. Monitoring systems have been set up to test various prototype constructions with satisfactory results. Yoon et al. [2] proposed a design procedure for multiple tube exchangers, considering thermal interference between neighbouring horizontal tubes with wide spacing in two rows. On the basis of numerical models they proposed a method for estimating heat transfer in a multi-tube system. Florides and Kalogirou [3] reviewed different calculation methods for underground exchangers. The use of these systems requires knowledge of the temperature profile in the soil at different depths. As climate conditions affect the temperature below the surface, usually the recommended depth for horizontal heat exchangers is more than 1.5-2 m.

De Paepe and Jannsens [4] propose a dimensioning method which considers a perfect contact between tube walls and soil. Then they assume that the tube wall temperature is constant. This model is appropriate for high thermal conductivity soils, ignoring the interference between tubes and assuming the convective internal resistance in the tube to be dominant. It is known, however, that tube arrays in which the outside heat transfer phenomenon is conduction, must be designed providing the tube spacing needed to minimize the decrease in energy available for heat transfer and the interference between tubes on a seasonal basis.

In this paper we will numerically study the heat transfer in horizontal tubes embedded in soil. Diameters from 50 to $200 \mathrm{~mm}$ are considered. For simplicity, we do not consider anisotropies or different materials around the tube. Seasonal analysis (3 months) will be made, in media in which the soil temperature has a very small variation along the year. By assuming a sufficient depth, the interaction of the site with the climate conditions can be ignored. For our 1D and 2D models we use the conditions of uniform outside tube wall temperature and uniform initial soil temperature. 
A 1D model for a single tube is studied first, characterizing the temperature field and heat transfer, and then a scaling law is proposed for conductance. 2D analyses for two parallel tubes are studied, and finally an analysis for a row of 9 tubes is made. The initial soil and tube wall temperatures are 288.15 and $298.15 \mathrm{~K}$ respectively in all tests.

\section{Definition of conductance}

To define the thermal conductance of soil around a tube we consider a single horizontal tube buried in a homogeneous isotropic soil of constant thermal conductivity which is initially at a uniform temperature, $T_{\infty}$. The temperature distribution in a region bounded internally by a cylindrical surface of radius $\mathrm{R}$ (tube) at prescribed surface temperature $T_{0}$, is given by [5]:

$$
\begin{aligned}
& \frac{\partial \theta}{\partial t}=\frac{\alpha}{r} \frac{\partial}{\partial r}\left(r \frac{\partial \theta}{\partial r}\right), \quad \theta(r, 0)=0, \\
& \theta(R, t)=\theta_{o}, \quad \theta(r, t) \rightarrow 0 \quad \text { as } r \rightarrow \infty
\end{aligned}
$$

where $\theta=T-T_{\infty}, \quad \theta_{o}=T_{0}-T_{\infty}$. The solution of this equation is

$$
\frac{\theta(r, t)}{\theta_{o}}=1+\frac{2}{\pi} \int_{0}^{\infty} e^{-\alpha u^{2} t}\left[\frac{J_{0}(u r) Y_{0}(u R)-J_{0}(u R) Y_{0}(u r)}{J_{0}{ }^{2}(u R)+Y_{0}^{2}(u R)}\right] \frac{d u}{u}
$$

The heat flow per unit area from the tube wall to the soil can be written as

$$
q=-k \frac{\partial \theta(R, t)}{\partial r}=\frac{4 \theta_{0} k}{R \pi^{2}} \int_{0}^{\infty} e^{-\alpha u^{2} t}\left[\frac{1}{J_{0}^{2}(u R)+Y_{0}^{2}(u R)}\right] \frac{d u}{u}
$$

The interface conductance, $\mathrm{h}$, of the soil around the tube, considering a constant temperature difference $\theta_{o}$ is defined as:

$$
h=\frac{q}{\theta_{o}}=\frac{4 k}{R \pi^{2}} \int_{0}^{\infty} e^{-\alpha u^{2} t}\left[\frac{1}{J_{0}^{2}(u R)+Y_{0}^{2}(u R)}\right] \frac{d u}{u}
$$

Although heat transfer around the tube is purely conductive, the conductance is analog to a convective coefficient, allowing the calculation of an overall heat transfer coefficient. From equation (5), the conductance depends on the thermal diffusivity and thermal conductivity of the soil, as well as the tube radius, and is expected to decrease in time. Eqn. (3) represents the temperature field around a single tube embedded in an infinite medium. Heat exchange between the tube 
and the medium will lower the temperature and the internal energy of the soil in the vicinity of the tube, so less energy will be available for collection as the season advances. As this effect will reduce the interface conductance, time scales for successful utilization of buried tubes have to be established. In the determination of heat transfer area for tube arrays in low enthalpy geothermal systems the presence of multiple tubes may affect the thermal conductance because of the mutual effect of neighboring tubes, which modify the temperature field around each tube. Such effects can only be described numerically.

\section{Numerical method}

Models were built using Comsol Multiphysics software, based on the finite element method. For a single tube a circular domain was used and rectangular domains for tube arrays. First the propagation distance of the tube wall thermal effect for long times had to be determined, to correctly define the size of the region. The correct diameter of the region is the one for which no effect of the tube temperature is observed at the frontiers of the domain at the end of the time interval. It may vary with the physical properties of the soil, as the temperature signal propagates farther in high thermal conductivity media.

Time intervals of three months were used to represent an entire season. An infinite region of analysis cannot be used in a numerical solution. To determine the region dimensions, tests using a region with adiabatic boundaries were used, in order not to force a particular temperature on the boundary. However, simulations accounting for the temperature recovery in the region are better done with an isothermal contour. It can be seen that if the region diameter is too small, an adiabatic boundary condition will cause underestimation of heat transfer by the tube, and the isothermal condition will overestimate this transfer. For the single tube, grids of at least 22368 triangular elements were used in a domain of $10 \mathrm{~m}$ in radius. The grids were adapted to the physics of the problem, concentrating nodes in the vicinity of the tube wall, to adequately represent high temperature gradients.

\section{Results}

\subsection{Single tube results: scaling laws for conductance}

Figure 1 shows radial temperature profiles in the first hours of operation of a single tube. A high temperature gradient is seen in the vicinity of the tube, but this gradient rapidly decreases in time, along with the thermal conductance.

The effect of soil properties is very important. It is customary that for every new project the thermal properties of the site are to be determined experimentally. In general the thermal conductivity of soils depends heavily on the moisture content. We consider representative of values for different types of soil. In simple cases as the single horizontal tube directly embedded in the soil, without intermediate material, the dependences of the conductance on the primary variables can be easily found from the results of simulation. 


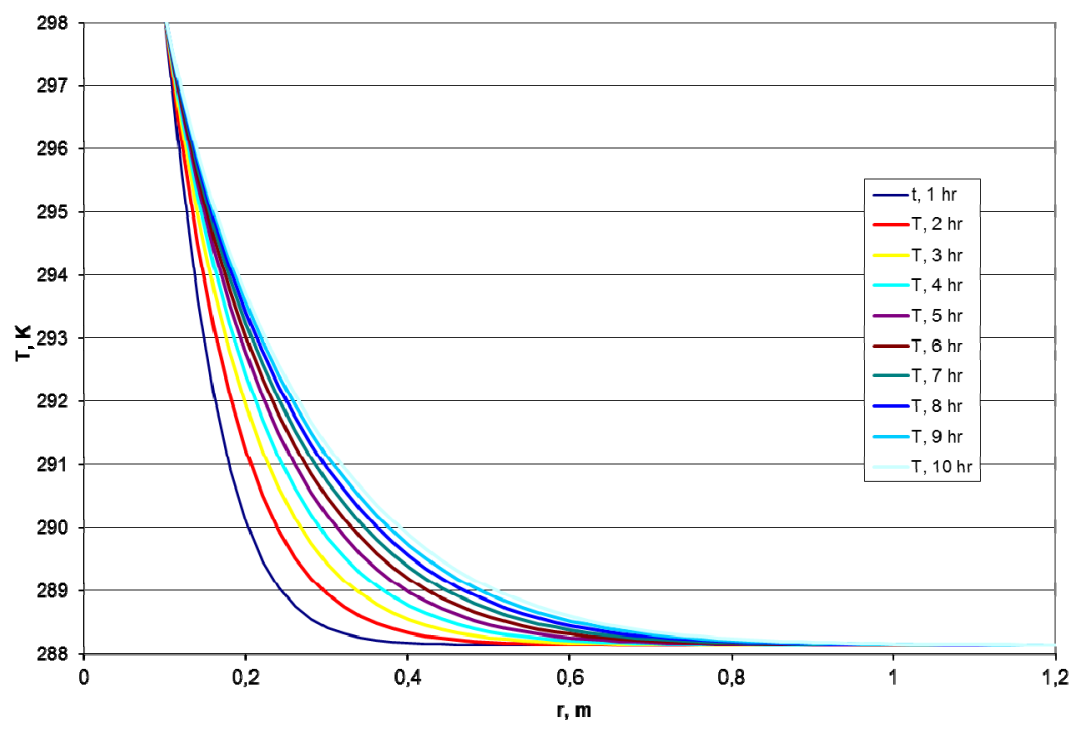

Figure 1: Initial decrease of radial temperature gradient for a single tube.

From 82 runs of the model for a single tube, the following expression was found:

$$
h \propto k \Delta t^{-0.14} R^{-0.696} \alpha^{-0.109}
$$

This scaling was obtained for radii between 1.5 and $24 \mathrm{~cm}$, time intervals of uninterrupted operation $(\Delta \mathrm{t})$ from 14 to 90 days, and thermal conductivities from 0.2 to $5 \mathrm{~W} / \mathrm{mK}$. These dependences agree essentially with the analytical form of the conductance for a single tube, eqn. (5).The most important single influences are that of the thermal conductivity of the soil and the tube diameter.

The soil thermal conductivity is the most important physical property affecting h, as shown by eqns. (5) and (6). This is confirmed in Figure 2, which shows that the thermal conductance reduces in time, reaching very low values at the end of a three month season. This is due to the progressive diminution of the normal temperature gradient on the tube surface as time advances (Figure 1), because of the modification of the local temperature field around the buried tube. This behavior of the conductance in time is similar to the one described analytically by Carslaw and Jaeger [5]. Their final values are rather modest, of about $2-4 \mathrm{~W} / \mathrm{m}^{2} \mathrm{~K}$.

Figure 2 includes the time averaged conductances over a 3 month time horizon. Although the average conductance is a conservative design parameter for the first month, in the next two months heat transfer will be lower than predicted by the average. Safety considerations may dictate the use of a value even lower than the time average in design, or to define shorter periods of use. 


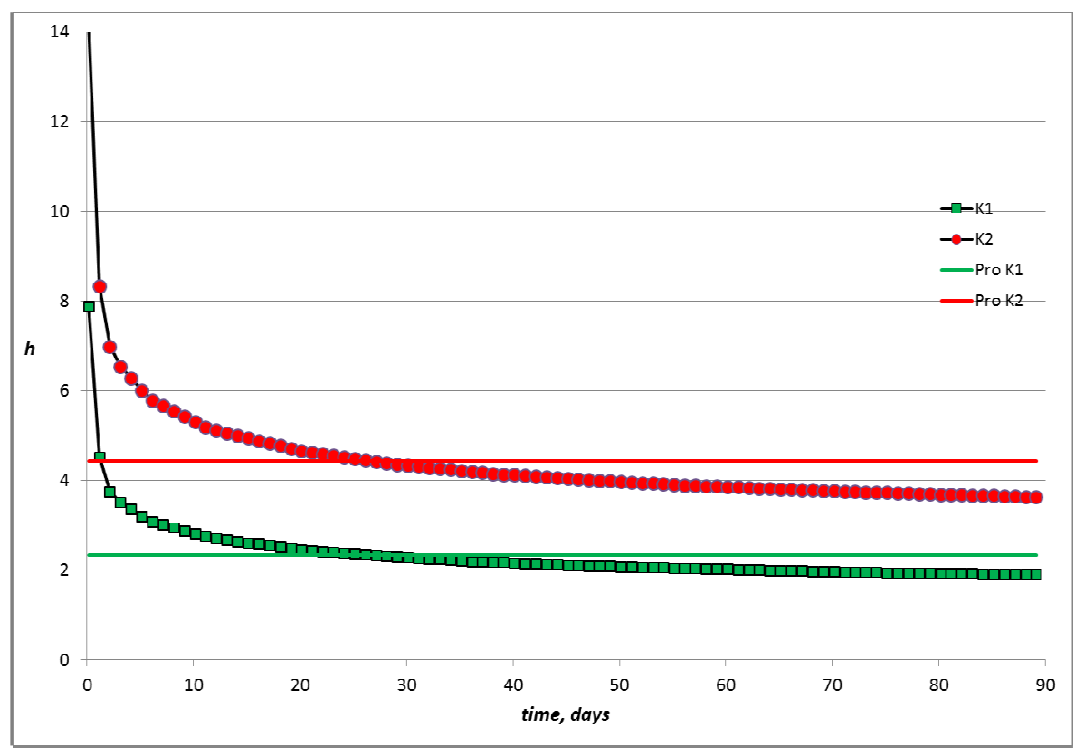

Figure 2: Conductance $\left(\mathrm{h}, \mathrm{W} / \mathrm{m}^{2} \mathrm{~K}\right)$ for soils of different properties: $\mathrm{K}_{2}$ : Soil of $\mathrm{k}=2 \mathrm{~W} / \mathrm{m} \mathrm{K}, \alpha=4.78 \times 10^{-7} \mathrm{~m}^{2} / \mathrm{s} . \mathrm{K}_{1}: \mathrm{k}=1 \mathrm{~W} / \mathrm{m} \mathrm{K}$, $\alpha=3.5 \times 10^{-7} \mathrm{~m}^{2} / \mathrm{s}$.

\subsection{Two tube results: thermal interference between tubes}

A 2D model for the cross section of two parallel horizontal tubes in a square region of side $12 \mathrm{~m}$ is analysed. The number of triangular elements in the region is 35680 . Results with $\mathrm{k}=1 \mathrm{~W} / \mathrm{m} \mathrm{K}$ and $\alpha=3.5 \times 10^{-7} \mathrm{~m}^{2} / \mathrm{s}$ are illustrated in Figures 3 and 4 . In this test the following values of tube spacing were used: 0.8 , $1,1.2,1.4,1.6,1.8$ and $2 \mathrm{~m}$. In Figure 3 the temperature field after three months shows a pronounced temperature increase in the region between the tubes. The normal temperature gradient varies along the tube perimeter, and is lower in the zone in which the tubes face each other. This will cause a reduced average conductance in a tube with respect to a single tube.

It can be seen that conductance is independent of spacing for short times (one day or less), and is nearly equal to the value for the single tube (Figure 4). This is because for short times the temperature variation caused by a tube in the soil has not yet reached the second tube, and therefore both tubes behave as single tubes. For longer times, interaction of the temperature fields, as exemplified in Figure 3, causes a lower conductance, especially a low spacing (Figure 4).

As an example, in this test the heat transferred by a tube is $7.62 \mathrm{~W} / \mathrm{m}$, while the other half transfers $5.59 \mathrm{~W} / \mathrm{m}$ only. The temperature increase in the region between tubes (which implies a lower effective temperature difference) is responsible for this diminution (see the temperature field in Figure 3). As conductance increases with tube separation, the single tube case is the upper limit of conductance for tubes assembled in pairs. 
Time $=7.704 \mathrm{e} 6$ Contour: Temperature $(\mathrm{K})$

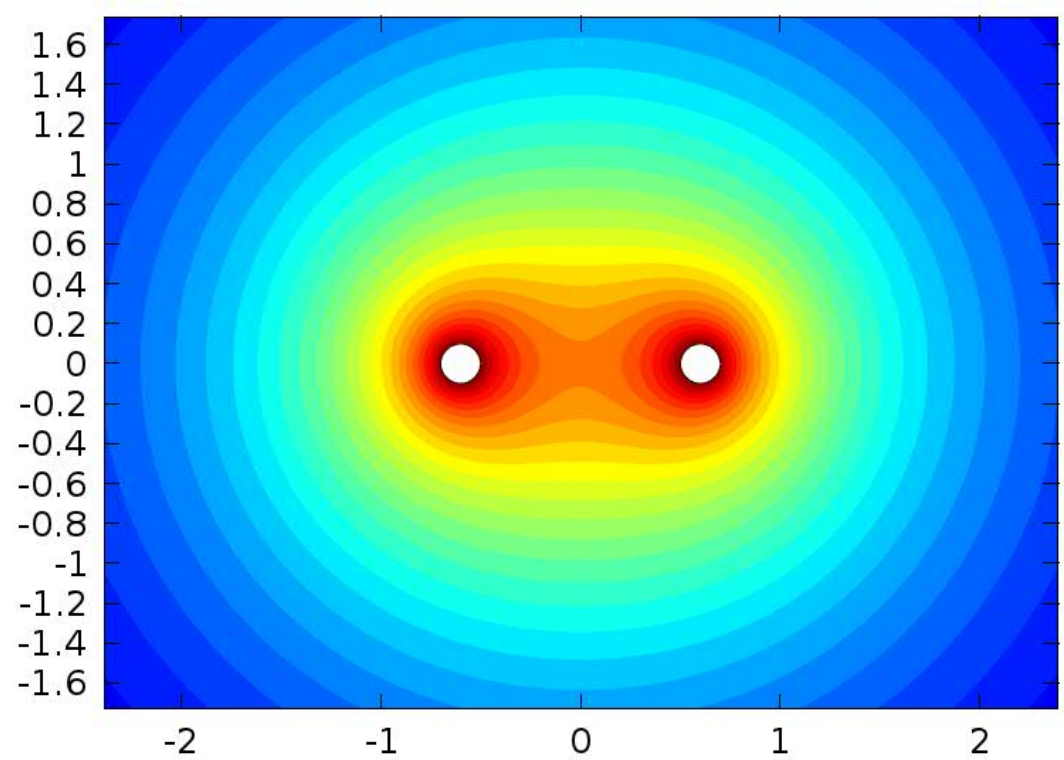

Figure 3: Temperature field around two parallel tubes after 90 days operation.

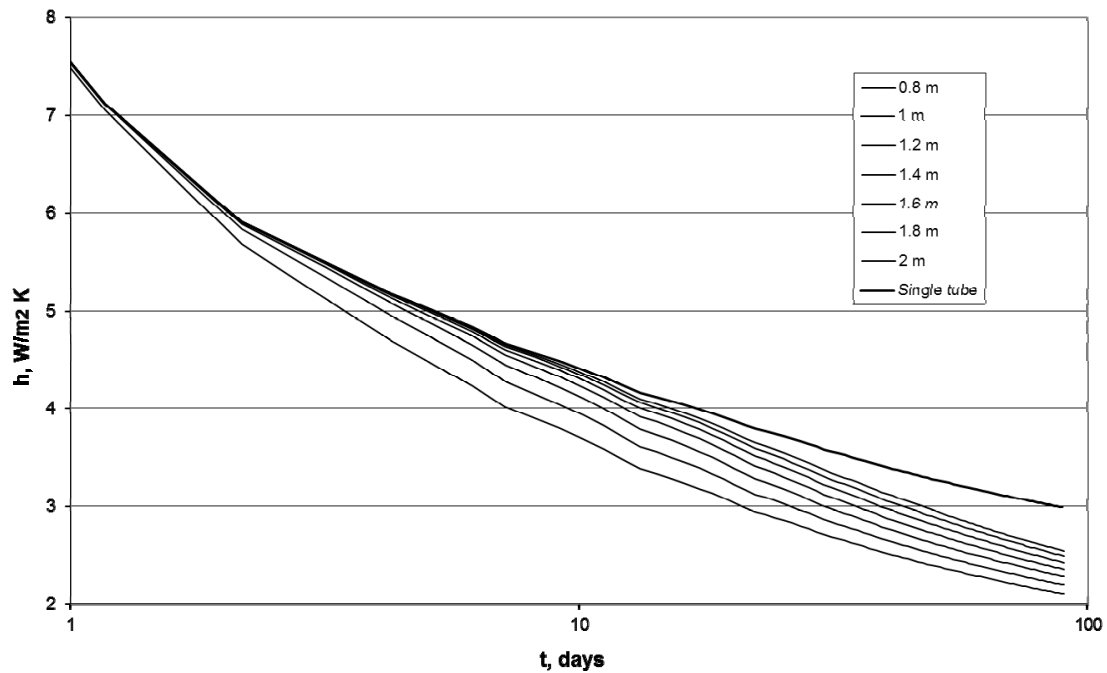

Figure 4: Two tubes: Small spacing has an adverse effect on conductance. 
To generalize these observations, we define the interference factor I (\%), which is defined as the heat transfer from a tube in a two tube array expressed as a percentage of the heat transfer for a single tube with all other conditions equal. This factor obviously depends on the tube radius, R, and on the tube spacing, S. From 49 runs the following empirical formula was obtained:

$$
I(\%)=\frac{3301+109.8 \times S}{63.19+R+S}
$$

In this formula $\mathrm{R}$ and $\mathrm{S}$ are expressed in centimetres. It can be seen that the interference is minimized for small radii and big distances. A similar index can be defined for the heat transfer from one tube in multiple tube arrays.

\subsection{Single line of tubes}

Consider a row of 9 horizontal tubes, with $\mathrm{k}=1 \mathrm{~W} / \mathrm{mK}$ and $\alpha=3.5 \times 10^{-7} \mathrm{~m}^{2} / \mathrm{s}$. The tube spacing is $1 \mathrm{~m}$. With this and other values of spacing the temperature in the region between tubes becomes very nearly uniform and similar to the tube wall temperature, after a time interval of three months (Figure 5). Therefore the heat transferred by each tube from the half perimeter facing the other tube will be very low. It is also apparent that even the sections of the perimeter not facing another tube directly are also affected by a low temperature gradient. Because of this, it becomes clear that the tube in a row will be even more ineffective than a tube in a two-tube array. Therefore, by extension of this reasoning, it is not recommendable to use two parallel rows of tubes in buried tube arrays, as it will create a hot isothermal zone between the rows. Also, with three rows the heat transferred by the inside row would be practically zero.

Now let us consider the row of tubes. The central tube is called T1, and the tubes located at his right side are successively termed T2, T3, T4 and T5. The conductance of these tubes are very different, as seen in Figure 6. This figure shows the evolution in time of the average conductances. The tube located in the extreme right show the highest conductances, while those in the center of the array exhibit the lowest heat transfer rates. This is explained by examining the temperature gradients around the different tubes in Figure 5.

\section{Conclusions}

A thermal conductance appropriate for use in the design of buried tube heat exchangers was proposed and described. Heat transfer models to characterize the thermal conductance of the soil around buried tubes and tube arrays were produced. The models reported consider the soil as a homogeneous, isotropic medium. Through the effect of local modification of temperature by the tubes the conductance decreases in time, with a significant variation in long periods. A scaling law is proposed to characterize this effect. Tube arrays have a poor heat transfer performance per tube with respect to a single tube. Favorable arrays consist of single rows of tubes with ample spacing. Conductance decreases 
appreciably from the ends of the row toward the centre. Although the axial variation of conductance in a subterranean heat exchanger would require three dimensional models, useful design criteria, based on 1D and 2D simulations have been proposed in this work.

Time $=7.704 \mathrm{e} 6$ Surface: Temperature (K)

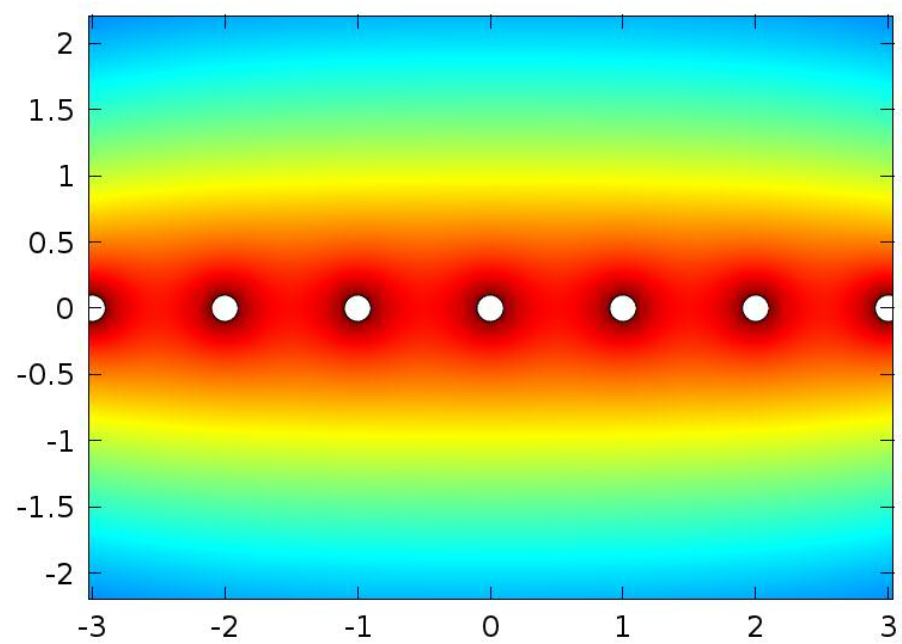

Figure 5: Temperature field around a line of 9 tubes at the end of three months.

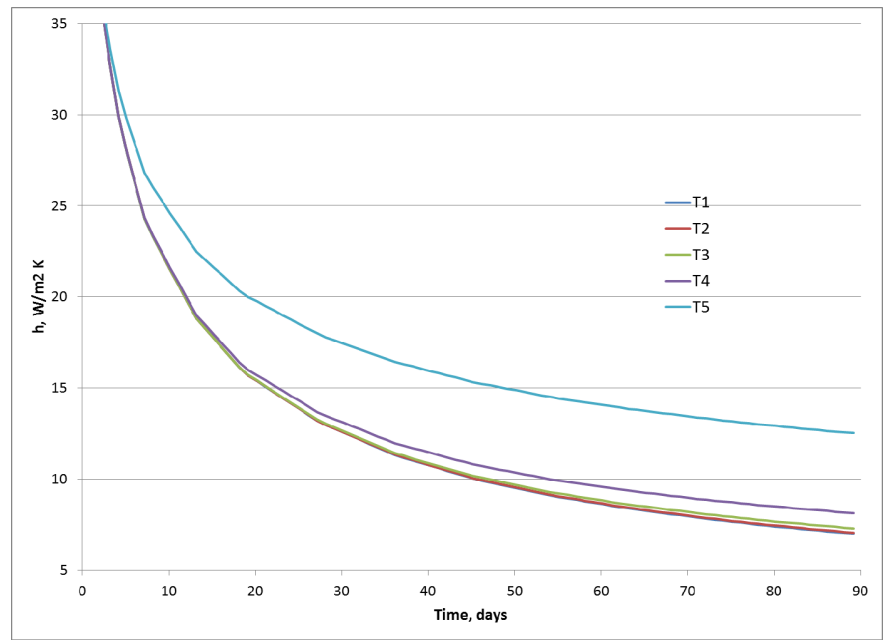

Figure 6: Conductance of different tubes in a 9 tube array. 


\section{References}

[1] Man, Y., Yang, H., Diao, N., Liu, J., \& Fang, Z., A new model and analytical solutions for borehole and pile ground heat exchangers. Int. $J$. Heat Mass Transfer, 53, pp. 2593-2601, 2010.

[2] Yoon, G., H. Tanaka, H. \& Okumiya, M., Study on the design procedure for a multi-cool/heat tube system, Solar Energy, 83, pp. 1415-1424, 2009.

[3] Florides, G. \& Kalogirou, S., Ground heat exchangers - A review of systems, models and applications, Renewable Energy, 32, pp. 2461-2478, 2009.

[4] De Paepe, M. \& Jannsens, A., Thermo-hydraulic design of earth-air heat exchangers, Energy and Buildings, 35, pp. 389-397, 2003.

[5] Carslaw, H.S., \& Jaeger, J.C., Conduction of heat en solids, $2^{\text {nd }}$ edition, Oxford University Press, Oxford, 1959. 